\title{
1.4 ASTHMA
}

Asthma is a chronic disease characterized by airway inflammation and reversible airflow limitation. It is one of the most common chronic conditions and it leads to marked morbidity and mortality in hospitalized patients. In the United States, 1 in 12 persons has asthma and nearly 50\% of affected individuals experience an asthma exacerbation each year, accounting for 1.8 million emergency department visits. $^{1,2}$ Annually, more than 400,000 hospital discharges occur with asthma as the primary diagnosis, with an average length of stay of 3.2 days. ${ }^{2}$ Hospitalists are central to the provision of care for patients with asthma through the use of evidence-based approaches to manage acute exacerbations and to prevent their recurrence. Hospitalists should strive to lead multidisciplinary teams to develop institutional guidelines and/or care pathways to improve efficiency and quality of care and to reduce readmission rates and morbidity and mortality from asthma.

\section{KNOWLEDGE}

Hospitalists should be able to:

- Define asthma and describe the pathophysiologic processes that lead to reversible airway obstruction and inflammation.

- Identify precipitants of asthma exacerbation, including environmental and occupational exposures.

- Recognize the clinical presentation of asthma exacerbation and differentiate it from other acute respiratory and nonrespiratory syndromes.

- Describe the role of diagnostic testing, including peak flow monitoring, used for evaluation of asthma exacerbation.

- Recognize indications for specialty consultation, including pulmonary and allergy medicine.

- Describe evidence-based therapies for the treatment of asthma exacerbations, which may include bronchodilators, systemic corticosteroids, and oxygen.

- Explain indications, contraindications, and mechanisms of action of pharmacologic agents used to treat asthma.

- Recognize signs and symptoms of impending respiratory failure.

- Explain the indications for invasive and noninvasive ventilatory support.

- List the risk factors for disease severity and death from asthma.

- Explain goals for hospital discharge, including specific measures of clinical stability for safe care transition.

\section{SKILLS}

Hospitalists should be able to:

- Elicit a thorough and relevant medical history to identify triggers of asthma and symptoms consistent with asthma exacerbation.

- Perform a targeted physical examination to elicit signs consistent with asthma exacerbation, differentiate find- ings from those of other mimicking conditions, and assess illness severity.

- Select appropriate diagnostic studies to evaluate severity of asthma exacerbation and interpret the results.

- Recognize indications for transfer to the intensive care unit, including impending respiratory failure, and coordinate intubation or noninvasive mechanical ventilation when indicated.

- Prescribe appropriate evidence-based pharmacologic therapies during asthma exacerbation, recommending the most appropriate route, dose, frequency, and duration of treatment.

- Communicate with patients and families to explain the natural history and prognosis of asthma.

- Facilitate discharge planning early during hospitalization.

- Develop an asthma action plan in preparation for discharge.

- Educate patients and families regarding the indications and appropriate use of daily use inhalers and rescue inhalers for asthmatic control.

- Ensure that patients receive training of proper inhaler and peak flow techniques before hospital discharge.

- Communicate with patients and families to explain symptoms and signs that should prompt emergent medical attention.

- Communicate with patients and families to explain the goals of care, including clinical stability criteria, the importance of preventive measures (such as smoking cessation, avoidance of second-hand smoke, appropriate vaccinations, and modification of environmental exposures), and required follow-up care.

- Communicate with patients and families to explain discharge medications, potential adverse effects, duration of therapy and dosing, and taper schedule.

- Document the treatment plan and provide clear discharge instructions for postdischarge clinicians.

- Provide and coordinate resources to ensure safe transition from the hospital to arranged follow-up care.

\section{ATTITUDES}

Hospitalists should be able to:

- Work collaboratively with primary care physicians and emergency physicians in making admission decisions.

- Employ a multidisciplinary approach, which may include pulmonary medicine, respiratory therapy, nursing, and social services, in the care of patients with asthma exacerbation.

- Follow evidence-based recommendations for the treatment of patients with asthma exacerbations.

\section{SYSTEM ORGANIZATION AND IMPROVEMENT}

To improve efficiency and quality within their organizations, hospitalists should: 
- Contribute to and/or develop educational modules, order sets, and/or pathways that facilitate use of evidence-based strategies for asthma exacerbation in the emergency department and the hospital, with goals of improving outcomes, decreasing length of stay, and reducing rehospitalization rates.

- Lead, coordinate, and/or participate in efforts to educate staff on the importance of smoking cessation counseling and other preventive measures.

- Lead, coordinate, and/or participate in multidisciplinary initiatives, which may include collaborative efforts with pulmonologists and respiratory therapists, to promote patient safety and optimize cost-effective diagnostic and management strategies for patients with asthma.

\section{Refrences}

1. Centers for Disease Control and Prevention. CDC Vital Signs: Asthma in the US. Available at: http://www.cdc.gov/vitalsigns/asthma/. Accessed June 2015.

2. Agency for Healthcare Research and Quality. Healthcare Cost and Utilization Project. U.S. Department of Health \& Human Services. Available at: http:// hcupnet.ahrq.gov/. Accessed June 2015. 